\title{
Switching performance of a polymer-stabilized antiferroelectric liquid crystal based on bent-core molecules
}

\author{
B. Atorf, ${ }^{1}$ A. Hoischen, ${ }^{1}$ M. B. Ros,${ }^{2}$ N. Gimeno, ${ }^{2}$ C. Tschierske,${ }^{3}$ G. Dantlgraber,${ }^{3}$ \\ and $\mathrm{H}$. Kitzerow ${ }^{1}$ \\ ${ }_{1}^{1}$ Department of Chemistry, University of Paderborn, Warburger Str. 100, 33098 Paderborn, Germany \\ ${ }^{2}$ Química Orgánica, Instituto de Ciencia de Materiales de Aragón-Facultad de Ciencias, \\ Universidad de Zaragoza, C.S.I.C. 50009 Zaragoza, Spain \\ ${ }^{3}$ Institute of Chemistry, Organic Chemistry, Martin Luther University Halle-Wittenberg, Kurt-Mothes-Str. 2, \\ 06120 Halle, Germany
}

(Received 26 February 2012; accepted 11 May 2012; published online 30 May 2012)

\begin{abstract}
Mixtures of a non-reactive and a photo-reactive liquid crystal, both consisting of bent core molecules, were used to prepare polymer-stabilized antiferroelectric liquid crystal cells by in-situ photo-polymerization. The mixtures exhibit a larger temperature range of the antiferroelectric phase than the pure compounds. The polymer network improves the ability of uniform alignment and thus enhances the switching contrast. In addition, the polymer-network reduces the switching times, in particular the relaxation time from the field-induced state to the ground state. (C) 2012 American Institute of Physics. [http://dx.doi.org/10.1063/1.4722794]
\end{abstract}

Ferroelectric ${ }^{1}$ and antiferroelectric liquid crystals ${ }^{2}$ are materials for display applications ${ }^{3}$ because of their bi-stable or tri-stable electrooptic switching behavior combined with fast switching speed. ${ }^{4,5}$ Generally, antiferroelectric liquid crystals show larger electrooptic switching angles of the optical axis than ferroelectric ones, which is advantageous to achieve high contrast ratios. ${ }^{6,7}$ Gel-like stabilization by a polymer network ${ }^{8,9}$ may be useful to adjust the electrooptic switching behavior, ${ }^{10-13}$ to overcome alignment problems, ${ }^{14}$ to stabilize a ferroelectric phase, ${ }^{15}$ or to enhance the switching speed. ${ }^{16,17}$ Chiral tilted smectic mesophases are known to exhibit sufficiently low symmetry elements to allow the appearance of polar properties, such as a local spontaneous polarization which results in ferroelectric or antiferroelectric behavior. ${ }^{4,5}$ Thus, chiral molecules are needed in the wellknown calamitic liquid crystals, which consist of rod-like molecules. However, particular interest in this research area was also focused on mesophases consisting of bent-core molecules. ${ }^{18,19}$ The latter can form chiral phases even when the molecules are non-chiral. ${ }^{20-23}$ Lamellar phases with tilted molecules of this kind show local phase chirality. Depending on the relative orientation of the local electric polarizations (parallel or antiparallel) and the relative molecular tilt directions of neighboring layers (synclinic or anticlinic), these phases can form paraelectric, ferroelectric, or antiferrolectric domains. According to further developing the promising switching performance, recent attempts were made to stabilize also the phases of bent-core molecules by fabricating network structures. ${ }^{24-26}$ Photo-reactive mesogenic monomers consisting of bent-core molecules were used to stabilize a conventional antiferroelectric calamitic liquid crystal. ${ }^{24}$ Reactive mesogens were also used to form bent-core elastomers. ${ }^{25}$ Also composites forming bent-core structures through hydrogen bonds ${ }^{26}$ have been investigated. However, there is a lack of investigations on polymer-stabilized liquid crystals, where both the non-reactive liquid crystal and reactive monomer consist of mesogenic molecules with a bent core. As a matter of fact, the concept of polymer-stabilized liquid crystals is known to be most effective when a nonreactive liquid crystal is mixed with a cross-linkable reactive mesogen exhibiting a very similar molecular structure and this mixture is photo-polymerized in-situ. ${ }^{8}$ For this purpose, the present paper describes electrooptic studies on polymerstabilized antiferroelectric liquid crystals that were fabricated in-situ from a very photo-stable non-reactive bent-core liquid crystal $^{27}$ and a bent-core photoreactive crosslinker. ${ }^{24,28}$

The samples investigated in this study are based on the non-reactive bent-core-mesogen (1ce), ${ }^{27}$ which exhibits on heating the phase sequence $\mathrm{Cr} 83^{\circ} \mathrm{C} \mathrm{SmCP}_{\mathrm{A}} 129^{\circ} \mathrm{C}$ Iso, where $\mathrm{SmCP}_{\mathrm{A}}$ denotes an antiferroelectric phase. This non-reactive liquid crystal was doped with the reactive methacrylate monomer $(\mathbf{M}){ }^{28}$ which also consists of bent-coremolecules and shows an antiferroelectric state. The synthesis of this compound is described in Ref. 28. Compound (M) shows the phase sequence $\mathrm{Cr} 85.9^{\circ} \mathrm{C} \mathrm{SmCP}_{\mathrm{A}} 108.6^{\circ} \mathrm{C}$ Iso. The concentration of the reactive compound in the mixtures varies in the range up to $3.26 \mathrm{wt}$. \%. A small amount (up to 1 wt. \%) of the photo-initiator Irgacure 651 (Ciba Geigy) was added in order to induce photo-polymerization through ultraviolet (UV) radiation. All compounds were diluted in dichloromethane. After mixing thoroughly, the solvent was evaporated.

Investigations of the electric and electro-optic properties were performed by means of standard ITO-test-cells with parallel alignment (EHC, Japan). A cell gap of $\mathrm{d}=\mathbf{2} \mu \mathrm{m}$ was used for optical switching experiments. However, cells with a thickness of $\mathrm{d}=\mathbf{1 5} \mu \mathrm{m}$ were utilized for measuring the spontaneous polarization $P_{s}$ in order to keep the bulk value of $\mathrm{P}_{\mathrm{s}}$ separate from surface polarizations. ${ }^{29}$ The samples were slowly cooled with a rate of $1{ }^{\circ} \mathrm{C} \mathrm{min}{ }^{-1}$ from the isotropic state to the antiferroelectric phase in a microscope hot-stage (Mettler, FP5). All sample preparations and the microscopic investigations on pristine samples were done under yellow light conditions to prevent undesired polymerization. The photo-polymerization was induced by UV irradiation using a metal halogen lamp (UV, type A). The distance between 
lamp and sample was about $40 \mathrm{~cm}$, yielding an intensity of $10 \mathrm{~mW} \mathrm{~cm}{ }^{-1}$ in the wavelength range from $320 \mathrm{~nm}$ to $400 \mathrm{~nm}$. The exposure times were $10 \mathrm{~min}$ or $20 \mathrm{~min}$ for samples with $\mathrm{d}=2 \mu \mathrm{m}$ and $\mathrm{d}=15 \mu \mathrm{m}$, respectively.

The electro-optic properties of both in the unpolymerized and polymerized state were examined in a polarizing stereo microscope (Leitz, type Wild M420). The triangularwave-method ${ }^{30}$ was used to measure the spontaneous polarization. For this purpose, a triangular voltage $\mathrm{V}_{0}(\mathrm{t})$ with a frequency of $20 \mathrm{~Hz}$ and an amplitude of $100 \mathrm{~V}_{0-\mathrm{pk}}$ was applied and the voltage drop $\mathrm{V}_{1}(\mathrm{t})$ across a constant resistor $(\mathrm{R}=1 \mathrm{k} \Omega)$ in series with the sample was recorded. This signal is proportional to the current through the sample. Ferroelectric or anti-ferroelectric switching of the cell results in one peak or two peaks of the current $\mathrm{I}(\mathrm{t})$ per cycle, which corresponds to the sign inversion or the induction of $\mathrm{P}_{\mathrm{S}}$, respectively. For an antiferroelectric sample, the area of one peak is proportional to the spontaneous polarization, $P_{s}=A^{-1} \int I(t) d t=A^{-1} R^{-1} \int V_{1}(t) d t$. The temperature dependence of the spontaneous polarization was measured in the temperature range from $120^{\circ} \mathrm{C}$ to $90^{\circ} \mathrm{C}$. The switching times were investigated by applying a rectangular voltage with an amplitude of $10 \mathrm{~V}_{0-\mathrm{pk}}$ (at a frequency of $1 \mathrm{~Hz}$ or 20 $\mathrm{Hz}$ ) over a cell gap of $2 \mu \mathrm{m}$. Temperatures larger than $120^{\circ} \mathrm{C}$ and electric field strengths larger than $20 \mathrm{~V} / \mu \mathrm{m}$ were avoided in order to prevent degradation of the compounds.

The experimental results of this study indicate both improved electro-optic performance and an enhancement of the temperature interval, where antiferroelectric switching is observed. The electro-optic switching behavior observed in a polarizing microscope reveals antiferroelectric switching behavior for compound (1ce) and its mixtures with compound (M), both in the pristine and in the photo-polymerized state. If the respective sample is placed between crossed polarizers and the azimuthal orientation of its optical axis at $\mathrm{V}_{0}=0$ is adjusted to be parallel to the plane of polarization of the incident light, the application of a voltage results in an increase of the transmitted intensity, independent on the sign of the voltage (Fig. 1). The example represented in Figure 1 shows light leakage in the field-off state, indicating that the uniformity of the alignment can still be improved. From the well-known relation for the transmitted intensity of uniaxial birefringent sample between crossed polarizers, $I=I_{\max }$ $\sin ^{2}(2 \varphi)$, where $\varphi$ is the azimuthal angle of the optical axis, one can estimate deviations of $\Delta \varphi \approx 16^{\circ}-17^{\circ}$ from the optimum alignment. The antiferroelectric origin of this switching

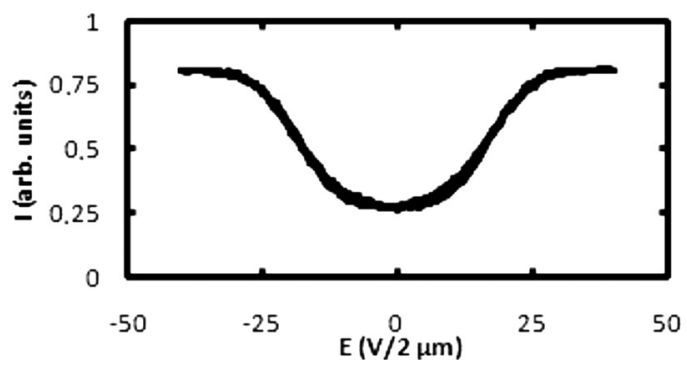

FIG. 1. Transmitted intensity versus field strength at $\mathrm{T}=100^{\circ} \mathrm{C}$ for a polymer-stabilized sample obtained from a mixture containing $98.0 \%$ (by weight) of compound (1ce), $1.5 \%$ of compound (M), and $0.5 \%$ photoinitiator.

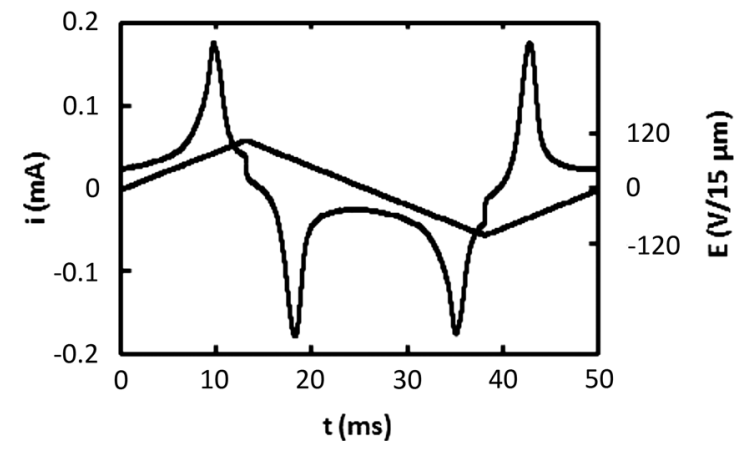

FIG. 2. Current through the same sample as represented in Fig. 1, measured at $\mathrm{T}=120^{\circ} \mathrm{C}$ when a triangular voltage (electric field amplitude $\mathrm{E}_{\max }=100$ $\mathrm{V}_{0-\mathrm{pk}} / 15 \mu \mathrm{m}$, frequency $\mathrm{f}=20 \mathrm{~Hz}$ ) is applied.

behavior is confirmed by the electric current that appears under the influence of a triangular voltage. On increasing voltage, a current peak appears (Fig. 2), which indicates a transition from the antiferroelectric state (electric polarization $\mathrm{P}=0)$ to a field-induced ferroelectric state $\left(\mathrm{P}=\mathrm{P}_{\mathrm{s}}\right)$. A subsequent current peak of opposite sign on decreasing voltage reveals the transition back to the antiferroelectric ground state.

The absolute value of the spontaneous polarization $\mathrm{P}_{\mathrm{S}}$ of the investigated system is quite large. Even close to the clearing temperature, it reaches values in the range of $\mathrm{P}_{\mathrm{s}} \approx 500-600 \mathrm{nC} / \mathrm{cm}^{2}$ (Table I). Comparison of two mixtures with different composition (Table I) indicates that the spontaneous polarization increases with increasing polymer concentration in the range of a few per cent polymer content.

Polymer-stabilization is also suitable to enhance the thermal stability of the antiferroelectric phase. The temperature interval of antiferroelectric switching increases by more than $10 \mathrm{~K}$. The pure compound (1ce) can be supercooled below the melting temperature $\left(83^{\circ} \mathrm{C}\right)$ and shows electrooptic switching in the temperature range from the clearing temperature down to approximately $70^{\circ} \mathrm{C}$. The clearing temperature is slightly lowered (down to $\approx 125-127^{\circ} \mathrm{C}$ ), when a few per cent of compound (M) are added. However, the clearing temperature increases again (to $\approx 129^{\circ} \mathrm{C}$ ) upon exposure to UV radiation. In the final polymer-stabilized mixtures obtained from mixtures of compound (1ce) with a few per cent of compound (M), crystallization is strongly suppressed, so that electro-optic switching is possible down to a temperature of $55^{\circ} \mathrm{C}$. In addition, the polymer network formed from a monomer content as low as $\mathrm{x}_{(\mathrm{M})}=1.5 \%$ facilitates a uniform alignment of the liquid crystal and results in a reasonable contrast ratio, which is larger than 8 (Table I). This contrast ratio decreases again on increasing polymer content, thereby reaching a value below 3 for $\mathrm{x}_{(\mathrm{M})}=3.26 \%$.

TABLE I. Spontaneous polarization and contrast ratio of polymer-stabilized mixtures made of $\left(99-\mathrm{x}_{(\mathrm{M})}\right)$ wt. \% compound $(\mathbf{1} \mathbf{c e}), \mathrm{x}_{(\mathrm{M})} \%$ compound $(\mathbf{M})$, and $1 \%$ Irgacure 651 at $\mathrm{T}=120^{\circ} \mathrm{C}$.

\begin{tabular}{lcc}
\hline \hline $\begin{array}{c}\text { Mass fraction } \mathrm{x}_{(\mathrm{M})}(\%) \\
\text { of compound }(\mathbf{M})\end{array}$ & $\begin{array}{c}\text { Spontaneous } \\
\text { polarization }\end{array}$ & $\begin{array}{c}\text { Contrast } \\
\text { ratio }\end{array}$ \\
\hline $1.50 \%$ & $452 \mathrm{nC} / \mathrm{cm}^{2}$ & 8.1 \\
$3.26 \%$ & $679 \mathrm{nC} / \mathrm{cm}^{2}$ & 2.6 \\
\hline \hline
\end{tabular}




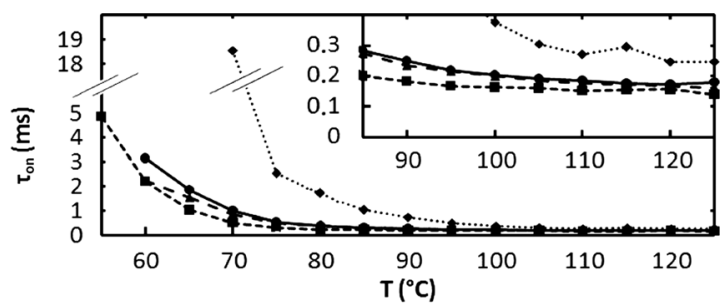

(a)

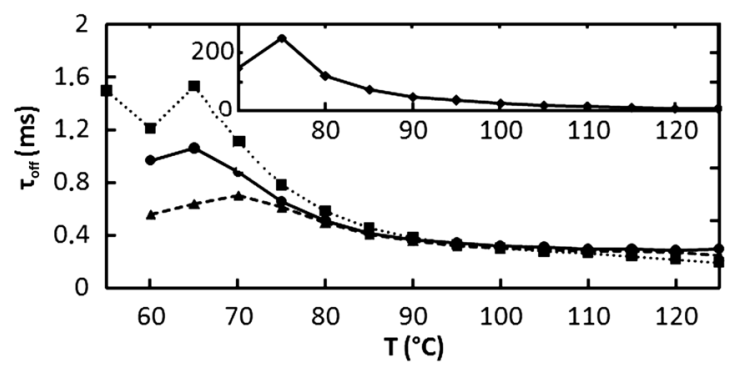

(b)

FIG. 3. Temperature dependence of the antiferroelectric switching times observed (a) when the field is switched on and (b) when the field is switched off, respectively. The different symbols correspond to polymer-stabilized

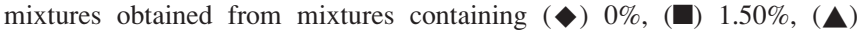
$2.43 \%$, and $(\bullet) 3.26 \%$ (by weight) of compound (M), respectively. The units of the axes in the insets of Fig. 3 are the same as indicated in the outer frames, i. e. $\left({ }^{\circ} \mathrm{C}\right)$ and $(\mathrm{ms})$, respectively.

Similarly low contrast ratios were obtained for samples containing the pure compound (1ce). In order to find the maximum contrast, the concentration needs to be studied more systematically.

The dynamics of the switching behavior shows the most remarkable influence of the polymer network on the switching behavior. The switching times were measured when the voltage was turned on (" $\tau_{\text {on }}$ ", Fig. 3(a)) and when the voltage

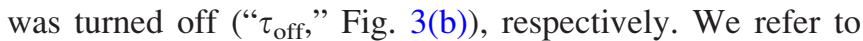
the latter time constants $\left(\tau_{\text {off }}\right)$ as "relaxation times". In all samples, the switching times decrease with increasing temperature. This can be attributed to a decreasing rotational viscosity on increasing temperature. For sufficiently large temperatures, the switching-on time $\tau_{\text {on }}$ and the switchingoff time $\tau_{\text {off }}$ are below $1 \mathrm{~ms}$ and below a few ms, respectively. In all cases, the polymer-stabilized samples show lower switching times than the pure compound (1ce). In particular, the relaxation time $\tau_{\text {off }}$ (Fig. 3(b)) is lowered, remarkably. At sufficiently high temperatures $\left(\mathrm{T} \approx 120^{\circ} \mathrm{C}\right)$, both time constants $\tau_{\text {on }}$ and $\tau_{\text {off }}$ reach values even smaller than $200 \mu \mathrm{s}$ in the mixture with a mass fraction $\mathrm{x}_{(\mathrm{M})}=1.5 \%$ of compound (M), while the respective time constants of the pure compound (1ce) are larger than $\tau_{\text {on }}=250 \mu \mathrm{s}$ and $\tau_{\text {off }}=9 \mathrm{~ms}$. In this elevated temperature range, the mixture with a mass fraction $\mathrm{x}_{(\mathrm{M})}=1.5 \%$ of compound $(\mathbf{M})$ shows the lowest switching times, while increasing the polymer content from $\mathrm{x}_{(\mathrm{M})}=1.5 \%$ to $\mathrm{x}_{(\mathrm{M})}=3.26 \%$ yields in larger switching times. So, similar to the influence of the composition on the contrast ratio, a modest polymer content $\left(\mathrm{x}_{(\mathrm{M})}=1.5 \%\right)$ is found to improve the switching behavior (larger contrast, higher switching speed), while considerably higher polymer contents are less advantageous.

In summary, the formation of polymer-stabilized antiferroelectric liquid crystals from a non-reactive liquid crystal and a reactive liquid crystalline monomer could be demon- strated for a system, where both components consist of bentcore molecules. In comparison with the pure non-reactive compound, the clearing temperature is slightly lowered, but the polymer network stabilizes the antiferroelectric $\mathrm{SmCP}_{\mathrm{A}}$ phase against crystallization, thereby enhancing the temperature range, where electrooptic switching is possible. The system shows high values of the spontaneous polarization, which increase with increasing polymer-concentration. The polymer network is also capable of improving the alignment, provided that the polymer content is limited. In addition to these advantages, the electro-optic switching times are considerably reduced in the polymer stabilized system. In particular, the reduction of the relaxation time is remarkable. In general, the switching-on speed of a liquid crystal can be improved by the amplitude of the applied electric field, while the switching-off speed is independent on the applied voltage and thus limits the performance of a liquid crystal light valve. Thus, the remarkable reduction of the relaxation time demonstrates not only that the antiferroelectric field-off is stabilized by the polymer network but may also be important for practical applications. However, the preliminary results presented here show also that the enhanced contrast and switching speed may decrease again if the polymer content becomes too large. Thus, the influence of the composition should be investigated more thoroughly.

Earlier investigations on polymer-stabilized antiferroelectric liquid crystals ${ }^{11}$ have shown that variations of the polymerization conditions open a lot of opportunities to modify the switching behavior and to optimize the performance for certain purposes. For example, in-situ-photo-polymerization under the influence of an applied electric field can stabilize a ferroelectric state rather than the antiferroelectric ground state, thereby inducing an asymmetric switching behavior or even a transformation from an antiferroelectric (tri-stable) to a ferroelectric (bi-stable) type of switching behavior. We expect that these opportunities are not limited to calamitic liquid crystals but can also be applied to polymer-stabilized liquid crystals made of bent-core molecules. Our preliminary results show that the samples investigated here represent an interesting material system for further developments in this direction.

The authors would like to acknowledge the financial support by the German Research Foundation (DFG, GRK 1464).

${ }^{1}$ R. B. Meyer, L. Liebert, L. Strzelecki, and P. Keller, "Ferroelectic liquid crystals," J. Phys. (Paris), Lett. 36, L69 (1975).

${ }^{2}$ S. Nishiyama, Y. Ouchi, H. Takezoe, and A. Fukuda, Jpn. J. Appl. Phys. 26, L1787 (1987).

${ }^{3}$ N. A. Clark and S. T. Lagerwall, Appl. Phys. Lett. 36, 899 (1980).

${ }^{4}$ J. W. Goodby, R. Blinc, N. A. Clark, S. T. Lagerwall, M. A. Osipov, S. A. Pikin, T. Sakurai, K. Yoshino, and B. Zeks, Ferroelectic Liquid Crystals Principles, Properties and Applications (Gordon and Breach, Philadelphia, PA, 1991).

${ }^{5}$ S. T. Lagerwall, Ferroelectric and Antiferroelectric Liquid Crystals (Wiley-VCH, Weinheim, 1999).

${ }^{6}$ K. D'havé, P. Rudquist, S. T. Lagerwall, H. Pauwels, W. Drzewinski, and R. Dabrowski, Appl. Phys. Lett. 76(24), 3528 (2000).

${ }^{7}$ S. T. Lagerwall, A. Dahlgren, P. Jägemalm, P. Rudquist, K. D'havé, H. Pauwels, R. Dabrowski, and W. Drzewinski, Adv. Funct. Mater. 11, 87 (2001).

${ }^{8}$ G. P. Crawford, S. Zumer, Liquid Crystals in Complex Geometries Formed by Polymer and Porous Networks (Taylor \& Francis, London, 1996). 
${ }^{9}$ I. Dierking, Polym. Chem. 2010, 1153-1159 (2010).

${ }^{10}$ J. Strauß and H.-S. Kitzerow, "Gray-scale in polymer-stabilized antiferroelectric liquid crystal displays," Appl. Phys. Lett. 69, 725 (1996).

${ }^{11}$ J. Strauss and H.-S. Kitzerow, Ber. Bunsenges. Phys. Chem. 102, 1609 (1998).

${ }^{12}$ J. Gloßmann, A. Hoischen, T. Röder, and H.-S. Kitzerow, Ferroelectrics 243, 95 (2000).

${ }^{13}$ M. C. Artal, M. B. Ros, J. L. Serrano, M. R. de la Fuente, and M. A. Pérez-Jubindo, Chem. Mater. 13, 2056 (2001).

${ }^{14}$ H. Furue and H. Yokoyama, Jpn. J. Appl. Phys., Part 1 42(9B), 6180 (2003).

${ }^{15}$ U. Singh and H. Gleeson, Mol. Cryst. Liq. Cryst. 439, 135 (2005).

${ }^{16}$ D. Engström, P. Rudquist, J. Bengtsson, K. D'havé, and S. Galt, Opt. Lett. 31(21), 3158 (2006).

${ }^{17}$ P. Rudquist, D. Elfström, S. T. Lagerwall, and R. Dabrowski, Ferroelectrics 344, 177 (2006).

${ }^{18}$ T. Niori, T. Sekine, J. Watanabe, T. Furukawa, and H. Takezoe, J. Mater. Chem. 6, 1213 (1996)

${ }^{19}$ T. Sekine, T. Niori, M. Sone, J. Watanabe, S. W. Choi, Y. Takanishi, and H. Takezoe, Jpn. J. Appl. Phys., Part 1 36, 6455 (1997).

${ }^{20}$ D. R. Link, G. Natale, R. Shao, J. E. Maclennan, N. A. Clark, E. Körblova, and D. M. Walba, Science 278, 1924 (1997).

${ }^{21}$ G. Heppke and D. Moro, Science 279, 1872 (1998).
${ }^{22}$ H. R. Brand, P. E. Cladis, and H. Pleiner, Eur. J. Phys. B 6, 347 (1998).

${ }^{23}$ R. Amaranatha Reddy and C. Tschierske, J. Mater. Chem. 16, 907 (2006).

${ }^{24}$ I. C. Pintre, J. L. Serrano, P. Hidalgo, M. B. Ros, F. Goc, and M. R. de la Fuenta, "Antiferroelectric liquid crystal gels based on a bentcore crosslinker," in 12th International Conference on Ferroelelectic Liquid Crystals, Zaragoza, Spain, 31st August-4th September 2009.

${ }^{25}$ R. Verduzco, P. Luchette, S. H. Hong, J. Harden, E. DiMasi, P. Palffy-Muhoray, S. M. Kilbey, S. Sprunt, J. T. Gleeson, and A. Jákli, J. Mater. Chem. 20, 8488 (2010).

${ }^{26}$ P.-J. Yang, L.-Y. Wang, C.-Y. Tang, and H.-C. Lin, J. Polym. Sci., Part A: Polym. Chem. 48, 764 (2010).

${ }^{27}$ G. Dantlgraber, D. Shen, S. Diele, and C. Tschierske, Chem. Mater. 14, 1149 (2002)

${ }^{28}$ J. Barberá, N. Gimeno, L. Monreal, R. Piñol, M. B. Ros, and J. L. Serrano, J. Am. Chem. Soc. 126, 7190-7191 (2004).

${ }^{29}$ S. Bawa, K. Saxena, and S. Chandra, "Surface and bulk switching dynamics in surface stabilized ferroelectic liquid crystals," Phys. Status Solidi A 114, 369 (1989).

${ }^{30}$ K. Miyasato, S. Abe, H. Takezoe, A. Fukuda, and E. Kuze, "Direct method with triangular waves for measuring spontaneous polarisation in ferroelectic liquid crystals,” Jpn. J. Appl. Phys., Part 2. 22(10), L661 (1983). 